\title{
SARS-CoV-2 interacts with platelets and megakaryocytes via ACE2-independent mechanism
}

Shu Shen ${ }^{1,2+}$, Jingyuan Zhang ${ }^{1+}$, Yaohui Fang ${ }^{1 \dagger}$, Sihong $\mathrm{Lu}^{3}$, Jun $\mathrm{Wu}^{3}$, Xin Zheng ${ }^{3^{*}}$ and Fei Deng ${ }^{1,2^{*}}$ (D)

\begin{abstract}
Evidence suggests that platelets may directly interact with SARS-CoV-2, raising the concern whether ACE2 receptor plays a role in this interaction. The current study showed that SARS-CoV-2 interacts with both platelets and megakaryocytes despite the limited efficiency. Abundance of the conventional receptor ACE2 and alternative receptors or co-factors for SARS-CoV-2 entry was characterized in platelets from COVID-19 patients and healthy persons as well as human megakaryocytes based on laboratory tests or previously reported RNA-seq data. The results suggest that SARS-CoV-2 interacts with platelets and megakaryocytes via ACE2-independent mechanism and may regulate alternative receptor expression associated with COVID-19 coagulation dysfunction.
\end{abstract}

Keywords: COVID-19, SARS-CoV-2, Platelets, Platelet activation, ACE2, Alternative receptors

\section{To the editor}

Associated with coagulative disorders, COVID-19 patients have increased platelet activation and aggregation, and platelet-monocyte aggregation [1-3], which highlights the critical role of platelets in SARS-CoV-2 infection and immunopathology [4]. Consistent with previous reports [1-3], our retrospective survey of plasma samples from a cohort of 62 cases (severe or fatal and moderate COVID-19 patients, Additional file 1: Table S1) showed that COVID-19 was associated with mild thrombocytopenia (platelet count $<150 \times 10^{9} / \mathrm{L}$ ) and increased thrombosis (elevated D-dimer levels), and patients had

\footnotetext{
*Correspondence: xinsunshine1011@aliyun.com; df@wh.iov.cn 'Shu Shen, Jingyuan Zhang and Yaohui Fang have contributed equally to this manuscript.

${ }^{1}$ State Key Laboratory of Virology, Wuhan Institute of Virology, Chinese Academy of Sciences, Hubei Province, Xiaohongshan 44\#, Wuchang District, Wuhan City 430071, People's Republic of China

${ }^{3}$ Department of Infectious Diseases, Union Hospital, Tongji Medical College, Huazhong University of Science and Technology, Hubei Province, Jiefang Avenue 1277\#, Jiangan District, Wuhan City 430022, People's Republic of China

Full list of author information is available at the end of the article
}

increased platelet activation (elevated sP-selectin and sGPVI levels) and cytokine (PF4 and RANTES) release upon platelet activation (Fig. 1a). Direct interaction of SARS-CoV-2 with human platelets was suggested based on increased P-selectin translocation on platelet surface (Fig. 1b), and elevated levels of GPVI, PF4, and RANTES in platelet culture supernatants (Fig. 1c). However, the characteristics and mechanisms of the direct interaction between SARS-CoV-2 and platelets are not well elucidated, and the role of platelet receptors in the interaction remains to be clarified $[4,5]$.

SARS-CoV-2 infection in human platelets and its progenitor megakaryocyte cell line MEG-01 in vitro was subsequently characterized. SARS-CoV-2 N expression was observed in some platelets and MEG01 cells (Fig. 2a). SARS-CoV-2 RNA was detected in both culture supernatant and MEG-01 cells after SARS-CoV-2 incubation and could be maintained with a slight increase until $48 \mathrm{~h}$ p.i. (Fig. 2b). This suggests that SARS-CoV-2 may infect and replicate in megakaryocytes despite insufficient efficiency. However, we failed to observe any viral particles in original author(s) and the source, provide a link to the Creative Commons licence, and indicate if changes were made. The images or other third party material in this article are included in the article's Creative Commons licence, unless indicated otherwise in a credit line to the material. If material is not included in the article's Creative Commons licence and your intended use is not permitted by statutory regulation or exceeds the permitted use, you will need to obtain permission directly from the copyright holder. To view a copy of this licence, visit http://creativecommons.org/licenses/by/4.0/. The Creative Commons Public Domain Dedication waiver (http://creativeco mmons.org/publicdomain/zero/1.0/) applies to the data made available in this article, unless otherwise stated in a credit line to the data. 


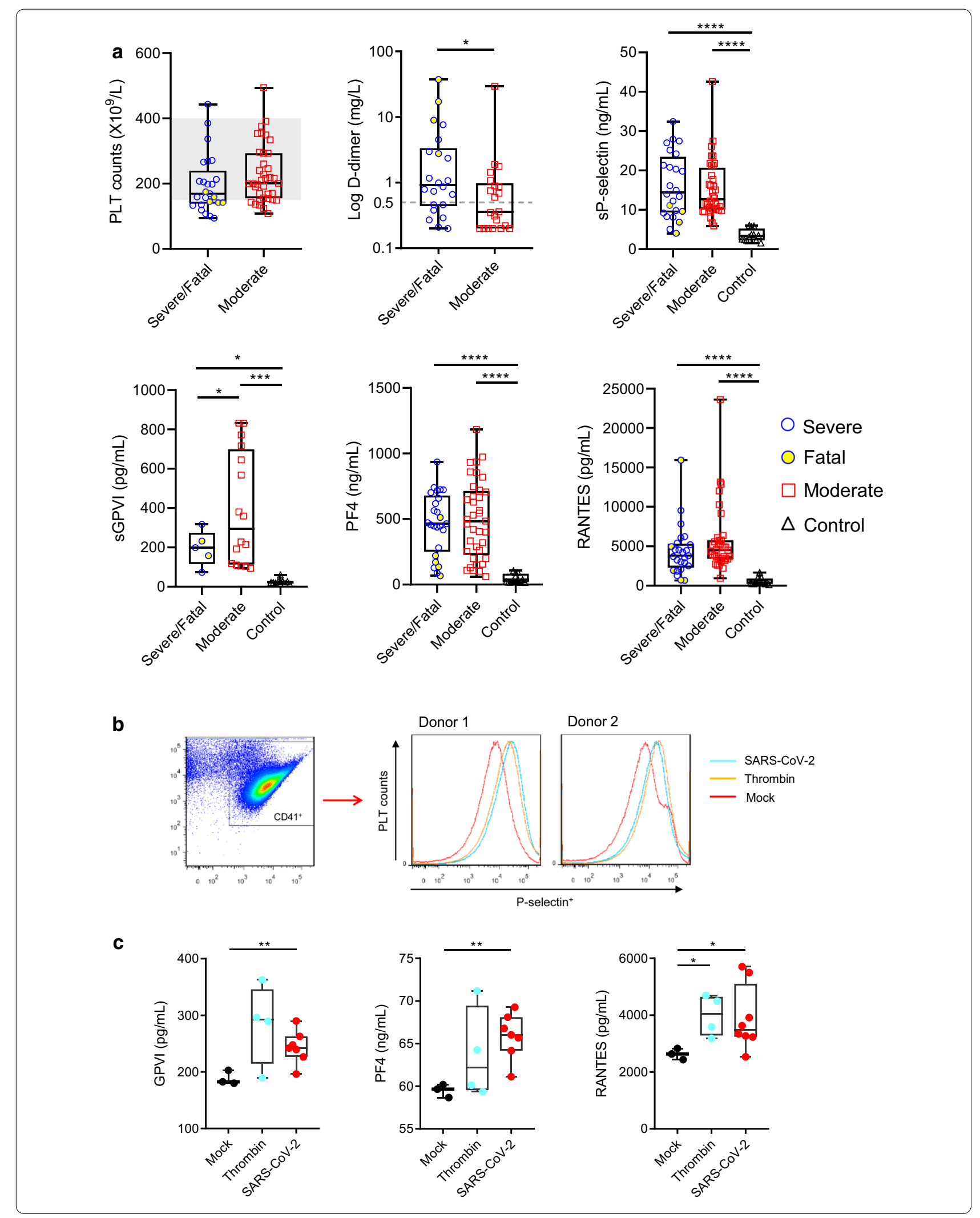




\section{(See figure on previous page.)}

Fig. 1 Increased platelet activation in COVID-19 patients and that stimulated by SARS-CoV-2. a Platelet counts and D-dimer levels of patients with severe/fatal and moderate COVID-19 are shown as the medians and interquartile ranges. The normal range of platelet count $\left(150-400 \times 10^{9} / \mathrm{L}\right)$ is shaded, and the upper limit value of D-dimer $(0.5 \mathrm{mg} / \mathrm{L}$ ) is indicated with dotted line. Soluble P-selectin levels (sP-selectin), soluble GPVI levels (sGPVI), PF4, and RANTES in plasma of patients with severe/fatal or moderate COVID-19, and healthy controls were measured through ELISA. b Platelet activation was investigated using platelets from healthy donors incubated with SARS-CoV-2, thrombin, or virus culture medium (Mock) for $3 \mathrm{~h}$ at $37^{\circ} \mathrm{C}$. P selectin surface translocation was measured using flow cytometry and results using platelets from two healthy donors are shown. $\mathbf{c}$ Levels of GPVI, PF4, and RANTES in the incubation supernatants were determined through ELISA. ${ }^{*}, \mathrm{P}<0.05$; $^{* *}, \mathrm{P}<0.01 ;{ }^{* * *}, \mathrm{P}<0.001 ;{ }^{* * *}, \mathrm{P}<0.0001$

MEG-01 cells through electron microscopy, probably because of using insufficient dose of viruses (1 MOI) for incubation, or limited infection in MEG-01 cells, as indicated by IFA images. SARS-CoV-2 RNA copies were lower in platelets $\left(10-10^{2}\right.$ copies $/ 10^{3}$ cells $)$ and culture supernatant $\left(10^{3}-10^{4}\right.$ copies $\left./ \mathrm{mL}\right)$, which diminished after $12 \mathrm{~h}$ (data not shown). Therefore, we speculate that platelets may not support SARS-CoV-2 replication. This echoes recent studies which have shown that SARS-CoV-2 entry in platelets may not be common in COVID-19 patients: SARS-CoV-2 RNA was detected in platelets from a few severe $(2 / 25$, $8 \%$ [2]; $2 / 11,18.2 \%$ [6]) and non-severe $(9 / 38,23.7 \%$ [6]) patients and was not detected in platelets from patients (0/24 [7]).

The evidence of direct interaction between SARS$\mathrm{CoV}-2$ and platelets or megakaryocytes raised the concern whether ACE2 plays a role in the process. The IFA and western blot assays showed a lack of ACE2 expression in both human platelets and megakaryocytes (Fig. 2c, d). The RNA abundance of 14 receptors or co-factors including ACE2 in human platelets and megakaryocytes was subsequently inspected based on RNA-seq data reported in previous studies [2, 8] (Additional file 1: Table S2 and S3). As summarized in Fig. 2e, the abundance order in platelets was: CD147 $>$ GRP78 $>$ KREMEN1 $>$ ADAM17 $>$ cathepsin $\quad$ L $>$ NRP1 $>$ ASGR1 $>$ CD209L/LSIGN $>$ CD301 $>$ CD26 $>$ CD206, but CD209/ DC-SIGN, ACE2, and TMPRSS2 were not identified.
Human megakaryocytes had similar receptor profiles, coupled with the detection of CD209/DC-SIGN. We also verified receptor abundance in MEG-01 and human platelets using qRT-PCR. In MEG-01 cells, CD147, GRP78, KREMEN1, cathepsin L, NRP1, and ASGR1 were detected, while in platelets, CD147, GRP78, KREMEN1, and ASGR1 were detected. ACE2 was not detected in MEG-01 cells or platelets (Fig. 2f). These results indicate that SARS-CoV-2 may use receptors other than ACE2 to interact with platelets or megakaryocytes.

Further analysis using the RNA-seq data showed unchanged GRP78, ADAM1, cathepsin L, GRP1, and ASGR1 abundance in platelets between ICU and nonICU COVID-19 patients and healthy persons and revealed elevated CD147 and KREMEN1 levels and reduced NRP1 levels in patients (Fig. 2g). This was also observed in MEG-01 cells with increased CD147 and KREMEN1 levels and slightly reduced NRP1 levels after SARS-CoV-2 incubation (Fig. 2h). These data suggest that SARS-CoV-2 infection may alter gene transcription in platelets and megakaryocytes, which is similar to DENV infection that markedly changes the platelet and megakaryocyte transcriptome [8].

Owing to their roles in binding to spike protein and facilitating virus entry [9-11], CD147, KREMEN1, and NRP1 triggering of SARS-CoV-2 entry in human platelets and megakaryocytes requires in-depth investigation. Moreover, based on the original functions of CD147 in signaling pathways via cell-cell interactions

\footnotetext{
(See figure on next page.)

Fig. 2 Characterization of SARS-CoV-2 interaction with human platelets and megakaryocytes. a IFA assays suggesting SARS-CoV-2 infection in platelets and megakaryocytes. Platelets from healthy donors and the megakaryocyte cell line MEG-01 were incubated with SARS-CoV-2 (1 MOI per test). SARS-CoV-2 N expression in platelets and MEG-01 cells were immunostained at $3 \mathrm{~h}$ p.i. and $24 \mathrm{~h}$ p.i., respectively. b Quantitative analysis of SARS-CoV-2 RNA copies in culture supernatants and in MEG-01 cells. $\mathbf{c}$ Immunofluorescence assay of ACE2 expression in MEG-01, platelets (PLT), Calu-3, Huh7, and 293 T cell lines. Bars, $10 \mu \mathrm{m}$. d Western blot analyses of ACE2 expression in cell lines including MEG-01, 293 T, HeLa, Huh7, and Calu-3, and platelets (PLT) from three healthy donors (D1, D2, and D3). Expression of $\beta$-actin in cells were blotted as inner control. e RNA transcripts of 14 receptors in human platelets and megakaryocytes were evaluated using bioinformatic methods using the RNA-seq data obtained from previous studies. $\mathbf{f}$ qRT-PCR detection of CD147, GRP78, KREMEN1, Cathepsin L, NRP1, ASGR1, and ACE2 in cell lines including Calu-3, Huh7, HeLa, 293 T, and MEG-01, and platelets from three healthy donors (D1, D2, and D3). The transcription levels were normalized to those of GAPDH in each of respective cell line or platelet samples and compared to MEG-01 or Calu-3 (shaded bars), as described in Additional file 1: Methods. g Comparison of CD147, KREMEN1, and NRP1 RNA levels in ICU and non-ICU COVID-19 patients with those in healthy persons using the RNA-seq data obtained from previous studies. $\mathbf{h}$ qRT-PCR detection of CD147, KREMEN1, and NRP1 transcription in MEG-01 cells after SARS-CoV-2 incubation
} 

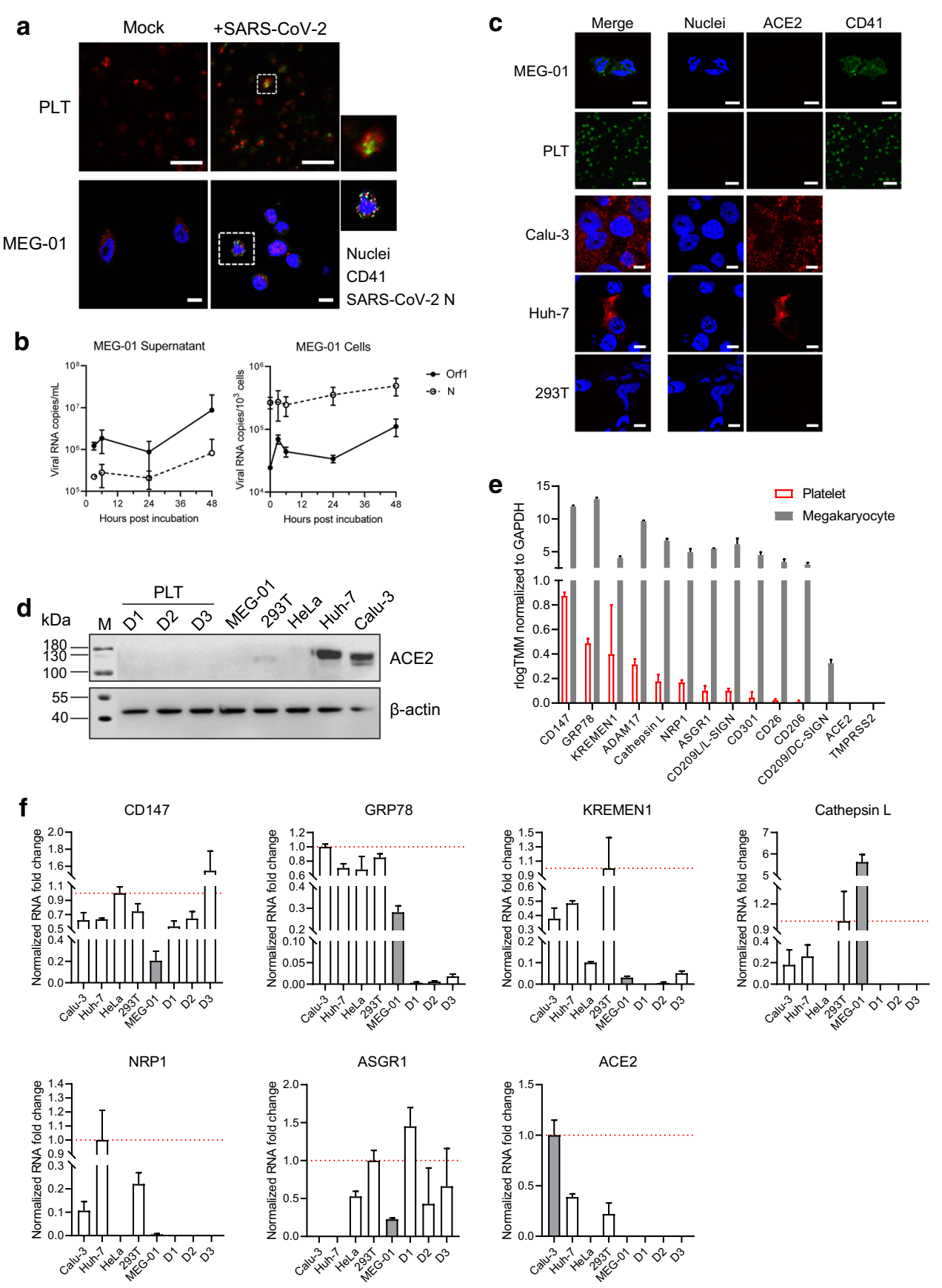

g
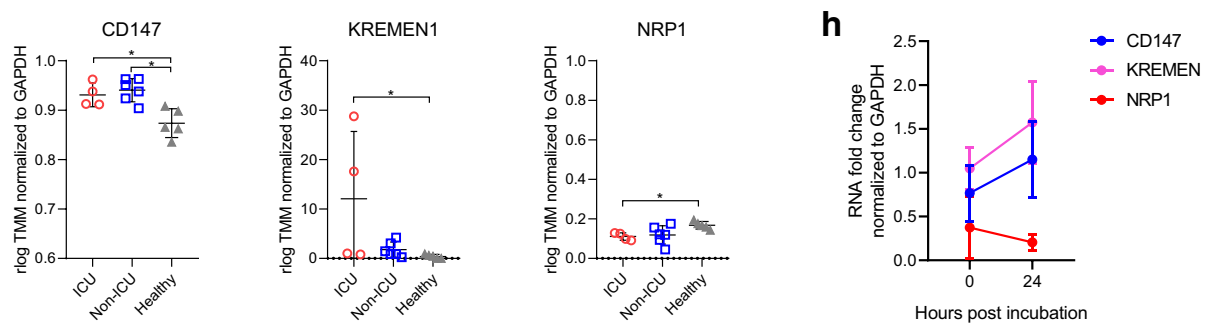
[9] and of NRP1 in cardiovascular, neuronal, and immune systems [10], SARS-CoV-2 interaction with platelets is suspected to regulate platelet-mediated immune response [12] and promote coagulation dysfunction in COVID-19 [10].

\section{Abbreviations}

COVID-19: Coronavirus disease 2019; SARS-CoV-2: Severe acute respiratory syndrome coronavirus; SARS-CoV-2 N: SARS-CoV-2 nucleoprotein; ACE2: Angiotensin-converting enzyme 2; SP-selectin: Soluble P-selectin; SGPVI: Soluble glycoprotein VI; PF4: Platelet factor 4; RANTES: C-C motif chemokine ligand 5, CXCL5; CD147: Basigin; GRP78: Glucose regulating protein 78; KREMEN1: Kringle containing transmembrane protein 1; ADAM17: A disintegrin and metalloproteinase 17; NRP1: Neuroplin-1; ASGR1: Asialoglycoprotein receptor 1; CD209L/L-SIGN: C-type lectin domain family 4, member M, CLEC4M; CD301: C-type lectin domain containing 10A, CLEC10A; CD26: Dipeptidyl peptidase 4, DPP4; CD206: Macrophage mannose receptor, MMR; CD209/ DC-SIGN: Dendritic cell (DC)-specific intracellular adhesion molecule 3 (ICAM3)-grabbing non-integrin; TMPRSS2: Transmembrane serine protease 2; DENV: Dengue virus; IFAs: Immunofluorescence assays; QRT-PCR: Quantitative reverse transcription-polymerase chain reaction; MOI: Multiplicity of infection; h p.i.: Hours post-inoculation.
\end{abstract}

\section{Supplementary Information}

The online version contains supplementary material available at https://doi. org/10.1186/s13045-021-01082-6.

Additional file 1. Detailed materials and methods, and supplementary tables and figure.

\section{Acknowledgements}

We acknowledge Mr. Ding Gao, Ms. Anna Du, Ms. Juan Min, Ms. Pei Zhang, and Ms. Bichao Xu from the Core Facility and Technical Support Facility of the Wuhan Institute of Virology for their technical assistance. We thank Mr. Jia Wu Mr. Hao Tang, and Mr. Jun Liu from the team of BSL-3 Laboratory of Wuhan Institute of Virology for their critical support in experimental activities, and Ms. Min Zhou and Mr. Zhong Zhang for their help with cell culture.

\section{Authors' contributions}

SS and DF designed and conceived the project. SS, ZJY, and FYH performed the research, analyzed the data, and wrote the manuscript. LSH, WJ, and ZX collected and analyzed the clinical data. All authors have contributed to and approved the manuscript.

\section{Funding}

This work was supported by the National Natural Science Foundation of China (U20A20135), the National Program on Key Research Project of China (2018YFE0200402, 2019YFC1200701, and 2020YFC0845801), and the Fundamental Research Funds for the Central Universities (2020kfyXGYJ016).

\section{Availability of data and materials}

All data generated or analyzed during this study are included in this published article (and its supplementary information files).

\section{Declarations}

Ethics approval and consent to participate

The study protocol was approved by the Ethics Committee of Tongji Medical College, Huazhong University of Science and Technology (number: 2020/0042-02-02)
Consent for publication

Not applicable.

\section{Competing interests}

The authors declare that they have no competing interests.

\section{Author details}

${ }^{1}$ State Key Laboratory of Virology, Wuhan Institute of Virology, Chinese Academy of Sciences, Hubei Province, Xiaohongshan 44\#, Wuchang District, Wuhan City 430071, People's Republic of China. ${ }^{2}$ National Virus Resource Center, Wuhan Institute of Virology, Chinese Academy of Sciences, 430071 Wuhan, People's Republic of China. ${ }^{3}$ Department of Infectious Diseases, Union Hospital, Tongji Medical College, Huazhong University of Science and Technology, Hubei Province, Jiefang Avenue 1277\#, Jiangan District, Wuhan City 430022, People's Republic of China.

Received: 19 February 2021 Accepted: 15 April 2021

Published online: 29 April 2021

\section{References}

1. Hottz ED, Azevedo-Quintanilha IG, Palhinha L, Teixeira L, Barreto EA, Pão CRR, et al. Platelet activation and platelet-monocyte aggregates formation trigger tissue factor expression in patients with severe COVID-19. Blood. 2020;136:1330-41.

2. Manne BK, Denorme F, Middleton EA, Portier I, Rowley JW, Stubben CJ, et al. Platelet gene expression and function in patients with COVID-19. Blood. 2020;136:1317-29.

3. Middleton EA, He XY, Denorme F, Campbell RA, Ng D, Salvatore SP, et al. Neutrophil extracellulartraps (NETs) contribute to immunothrombosis in COVID-19 acute respiratory distress syndrome. Blood. 2020;136:1169-79.

4. Koupenova M, Freedman JE. Platelets and COVID-19: Inflammation, hyperactivation and additionalquestions. Circ Res. 2020;127(11):1419-21.

5. Campbell RA, Boilard E, Rondina MT. Is there a role for the ACE2 receptor in SARS-CoV-2 interactions with platelets? J Thromb Haemost. 2021;19(1):46-50.

6. Zaid Y, Puhm F, Allaeys I, Naya A, Oudghiri M, Khalki L, et al. Platelets can associate with SARS-Cov-2 RNA and are hyperactivated in COVID-19. Circ Res. 2020;127(11):1404-18.

7. Bury L, Camilloni B, Castronari R, Piselli E, Malvestiti M, Borghi M, et al. Search for SARS-CoV-2 RNA in platelets from COVID-19 patients. Platelets. 2021;32(2):284-7.

8. Campbell RA, Schwertz H, Hottz ED, Rowley JW, Manne BK, Washington $A V$, et al. Human megakaryocytes possess intrinsic antiviral immunity through regulated induction of IFITM3. Blood. 2019:133(19):2013-26.

9. Wang K, Chen W, Zhang Z, Deng Y, Lian JQ, Du P, et al. CD147-spike protein is a novel route for SARS-CoV-2 infection to host cells. Signal Transduct Target Ther. 2020;5(1):283.

10. Mayi BS, Leibowitz JA, Woods AT, Ammon KA, Liu AE, Raja A. The role of Neuropilin-1 in COVID-19. PLOS Pathog. 2021;17(1):e1009153.

11. Gu Y, Cao J, Zhang $X$, Gao H, Wang Y, Wang J, et al. Interaction network of SARS-CoV-2 with host receptome through spike protein. bioRxiv-Microbiol;2020. https://doi.org/10.1101/2020.09.09.287508

12. Savla SR, Prabhavalkar KS, Bhatt LK. Cytokine storm associated coagulation complications in COVID-19 patients: Pathogenesis and Management. Expert Rev Anti Infect Ther. 2021. https://doi.org/10.1080/14787210.2021. 1915129

\section{Publisher's Note}

Springer Nature remains neutral with regard to jurisdictional claims in published maps and institutional affiliations. 